\title{
A Case of "Ifufunyane": A Xhosa Culture-Bound Syndrome
}

Case presented by: Dana J. H. Niehaus, Dan J. Stein, Liezl Koen, Christine Lochner, Jacqueline E. Muller, N. Irene Mbanga and Robin A. Emsley: Department of Psychiatry, University of Stellenbosch, South Africa.

STEIN: Department of Psychiatry, University of Cape Town, South Africa.

Case discussion: Jack M. Gorman MD: Department of Psychiatry, Mount Sinai School of Medicine, New York.

Clinicians and patients frequently have a different understanding and interpretation of the nature of an illness. While many reasons for these discrepancies can be postulated, differences in sociocultural background often play an important role-especially in the field of psychiatry. At our tertiary psychiatric hospital in South Africa, where standard Western teachings are followed, clinicians are often confronted by patients who have a markedly different interpretation of their psychiatric symptoms compared with the clinician's perspective. For instance, "ifufunyane" (plural "amafufunyana"), a ritualized" "possession state," often thought to result from witchcraft, is frequently reported by South African (Xhosa) patients with psychosis (including schizophrenia) and their families.

This condition, which was not included as a culture-bound syndrome in the Diagnostic and Statistical Manual of Mental Disorders, 4th edition, is characterized by a confused mental state 5 and was originally described as a self-contained "hysterical condition characterized by people speaking in a strange muffled voice in a language that cannot be understood (speaking in tongues), and strange and unpredictable behaviour." However, recent work suggests that "ifufunyane" may cover a range of different disorders and phenomena including trance states (diagnosed in DSMIV as dissociative disorders), aggressive behavior, and psychomotor agitation. Against this background, we present a case study that illustrates, first, a typical case of "ifufunyane" in a patient with schizophrenia and, second, the divergence of western and Xhosa cultural interpretations thereof. Some of the implications of this divergence are discussed.

\section{Initial case presentation}

The patient is an African (Xhosa) male from South Africa who presented for the first time at age 23 to our tertiary psychiatric hospital with a history of several months of auditory hallucinations, paranoid delusions, and agitation. The patient lived in a shack in a shantytown with his family and worked as a cleaner in a state hospital; although poor, he had sufficient income to make ends meet. There had been a gradual deterioration in the patient's functioning over several months, and he was diagnosed with schizophrenia by a psychiatrist at the time of his initial presentation.

\section{Treatment}

The patient was admitted to the hospital, where treatment with oral antipsychotics was initiated. Treatment was continued for 4 weeks, at which time the symptoms were sufficiently resolved for the patient to be discharged to the care of his family. Both the patient and the family believed that he was suffering from "ifufunyane." His family had previously taken the patient to a traditional healer for herbal treatment and, after his discharge, the patient discontinued the antipsychotics in favor of these agents.

The patient's symptoms again worsened and a month after being discharged from the hospital, he required readmission. Oral antipsychotic medications were again prescribed with success. Hospital staff, the patient, and his family then met to discuss a future management strategy. The patient and 
his family were given the opportunity to describe their view of the cause of symptoms and their ideas concerning the optimal treatment (i.e., that the patient had a form of possession that could be treated with herbal methods and religious rituals). Hospital staff in turn emphasized that in their view the patient was suffering from a psychiatric disorder and that ongoing antipsychotic medication was necessary. The patient and family agreed to continue to use antipsychotics.

\section{Follow-up}

The patient has remained compliant with medication since that time (he is now 39) and has also remained reasonably functional, albeit unemployed. However, upon questioning, he admitted to being somewhat distressed about the fact that his auditory hallucinations have never entirely disappeared. He has visited various traditional healers on a number of occasions to try to obtain further help and clarify the cause of his symptoms. Most recently, a traditional healer informed him that he did not have "ifufunyane" - rather, the healer told him that his ancestors were scolding him (in the form of voices) and that this was a punishment for not having fulfilled certain customary rituals (e.g. sacrifice of a cow). He was instructed to perform these rituals and complied with this instruction.

The patient himself remains ambivalent about whether the ongoing voices are "ifufunyane" or the scolding of ancestors. He is clear that the voices are "bad" and explains that the acts that they instruct him to perform (e.g., killing people) are immoral. Furthermore, he is committed to continuing to take his medication because he feels the voices would be worse without the medication and also that it helps him to sleep.

\section{Comment}

From a clinical point of view, patients who hold an explanatory model of their illness (i.e., causes and treatment) that is entirely different from that of the clinician will presumably have a poorer prognosis. In particular, as exemplified in this case, such patients may be nonadherent with prescribed treatment or may attempt to adhere to both traditional (mostly herbal) treatment and antipsychotic medication. In order to improve adherence to standard treatment, it may be necessary to negotiate a shared explanatory model.8 Fortunately, "ifufunyane" shares a number of features with a biomedical model. In both cases, the person with symptoms is seen as a victim in need of help, a pathogenic neuropsychiatric process is postulated, and pharmacological methods are used to reverse these. The two paradigms may therefore not be "incommensurable."

In the current case, successful negotiation of a shared explanatory model was in fact undertaken, and the patient continues to operate with multiple explanatory models of his symptoms ("ifufunyane," ancestor scolding, biomedical). While this causes him some confusion with regard to a theoretical explanation for his symptoms, at a practical level the patient has remained adherent to medication for nearly two decades and continues to function reasonably independently in the community. While not all Western diagnostic categories and African culture-bound syndromes may be commensurable, the explanatory model of "ifufunyane" overlaps in certain important respects with that of schizophrenia. It is therefore possible to negotiate a shared explanatory model with patients with "ifufunyane" in which the use of antipsychotics is given a central place. The case report presented here demonstrates that patients who perceive themselves as having "ifufunyane" but who suffer from classical symptoms of schizophrenia may respond well to negotiation of a shared explanatory model and to treatment with antipsychotics. Similar negotiations play a crucial role in successful treatment even when clinician and patient have similar sociocultural backgrounds but differ in their explanatory models of a disorder. 


\section{Discussion by Jack M. Gorman, MD}

How flexible should we be when confronted with challenges to Western devotion to the "scientific method?" Since tolerance is generally seen as a virtue, most physicians would probably answer that we should be very open to ideas that are not immediately consonant with ours. We can appreciate, however, that sometimes this gets out of hand. Today, for example, we are confronted with a concept known as "intelligent design," which is essentially a challenge to the inductive method of scientific reasoning that has become the foundation for our modern understanding of the natural and physical world. The inductive method clearly supports Darwinian evolution. In our wish to be understanding of alternative views, it is incumbent upon those of us who abide by science not to lose our scientific compass. Similarly, the case wonderfully described by Dr. Niehaus and colleagues presents an interesting challenge to those of us trained in Western medicine, although here the issues here are not nearly so clear-cut as adjudicating between natural selection and intelligent design.

The patient and his family are not versed in Western psychiatry and, when bizarre behavior strikes, they ascribe it to witchcraft, as is their tradition. On the one hand, we do not want to deny this man the benefits of modern biomedical science in approaching what is clearly a case of schizophrenia. On the other hand, we do not want to seem arrogant or elitist. "Ifufunyane" is a type of religious belief among his family and we are inclined to be respectful of these ideas that may be unfamiliar to us. How do we proceed? It is important to realize that when it comes to religion, psychiatry is generally on shaky ground. Freud professed to have no knowledge of religion and wrote about it with hostility. To him, religious belief was a form of neurosis. Not wishing to seem bigoted, he directed most of his antagonism toward his own religion, including proposing the somewhat extreme premise in Moses and Monotheism that Moses was murdered by the Jewish people in the Sinai desert and the body hidden.

This set the stage for a longstanding disregard of religious belief by psychiatry, something that has only recently been tackled as a major shortcoming of our field. Hence, it is refreshing indeed to see the deft way in which Dr. Niehaus and colleagues handled the situation with their patient. Taking seriously the concept of "ifufanyane," they brokered an arrangement that allows the family to simultaneously maintain their religious beliefs and encourage the patient to adhere to a regimen of antipsychotic medication. This has worked to the degree that the patient has remained engaged in therapy for many years and takes his medication and, equally important, the family is not made to feel alienated or belittled. This is psychiatry practiced at its highest level as an art and as science. There are two other sobering lessons that we can also learn from this case. First, it is clear that the patient and his family are impoverished. With all of our current emphasis on the molecular genetics of schizophrenia, it is important to remember that strong, empirically supported risk factors for schizophrenia are social adversity and economic disadvantage. Although many would have us believe that this is due to "social drift" - schizophrenia forces people into lower socioeconomic strata - other convincing data suggest that social adversity may play an etiological role in the pathogenesis of schizophrenia schizophrenia.

Thus, we are again reminded that our field must deal with social issues in order to understand the disorders for which we are responsible. Second, although it is nice to hear that the patient in this case adhered to medication and did relatively well, he did not become entirely free of psychotic symptoms and was not able to sustain employment. Thus, despite having knowledgeable and caring physicians, the ravages of schizophrenia have not been entirely abated for him. This is characteristic of medication response in patients with schizophrenia. Antipsychotic drugs clearly work, but complete remission is uncommon. As the recently published CATIE results document, 11 drugs for schizophrenia are difficult for patients to accept and do not always work. In such cases, whether in the Western world or not, the presence of an accepting family willing to work hard on behalf of the patient is invaluable. There is, therefore, a lot we can learn from this patient and his family. Perhaps it is they who are exhibiting tolerance for us? 


\section{References}

Robertson BA, Kottler A. Cultural issues in psychiatric assessment of Xhosa children and adolescents. South African Medical Journal 1993; 83:207-8.

Mbanga I, Niehaus DJH, Stein DJ. Attitudes towards and beliefs about schizophrenia in Xhosa families with affected probands. Curationis 2002:69-74.

Swartz L. Culture and mental health. Cape Town: Oxford University Press; 1998.

American Psychiatric Association. Diagnostic and statistical manual of mental disorders, 4th edition. Washington, DC: American Psychiatric Association; 1994.

Bührmann MV. Living in two worlds. Cape Town: Human and Rousseau; 1984.

Bührmann MV. Lifestyle and disease: Thwasa and bewitchment. S Afr Med J 1982; 61:877-9.

Koen L, Niehaus D, Laurent C, et al. Traditional treatment methods. S Afr Med J 2003;93:443.

Kleinman A. Rethinking psychiatry: From cultural category to personal experience. New York: Free Press; 1988.

Kuhn TS. The structure of scientific revolutions, 2nd ed. Chicago: University of Chicago Press; 1971.

Wicks S, Hjern A, Gunnell D, et al. Social adversity in childhood and the risk of developing psychosis: A national cohort study. Am J Psychiatry 2005;162:1652-7.

Lieberman JA, Stroup TS, McEvoy JP, et al. Effectiveness of antipsychotic drugs in patients with chronic schizophrenia. N Engl J Med 2005;353:1209-23. 